\title{
A Rare Case of Chronic Primary Angle-Closure Glaucoma in a Young Woman with Coexisting Pathologic Myopia
}

\author{
Authors: \\ Ni Putu Ayu Reza Dhiyantari* ${ }^{*}$ \\ Nurwasis ${ }^{\circ}$ \\ Evelyn Komaratih \\ Yulia Primitasari ${ }^{\circledR}$ \\ Affiliations: \\ Department of Ophthalmology, \\ Faculty of Medicine, Universitas \\ Airlangga-RSUDDr. Soetomo \\ Surabaya, Indonesia.
}

Corresponding author: Ni Putu Ayu Reza Dhiyantari ni.putu.ayu-2019@fk.unair.ac.id

Dates:

Received: 16 July 2021

Revised: 27 September 2021

Accepted: 29 September 2021

Published: 03 November 2021

DOI:

https://doi.org/10.20473/

vsehj.v1i1.2021.10-16

\section{Copyright:}

(C) 2021 Author(s). Open access under Creative Commons Attribution-Share Alike $\quad 4.0 \quad$ International Licence (CC-BY-SA).

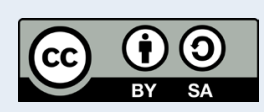

\begin{abstract}
Introduction: Primary Angle-Closure Glaucoma (PACG) is usually present in adults older than forty and is more common in hyperopic eyes. Angle-closure is usually related to structural or developmental ocular abnormalities in young individuals. Case presentation: We presented a rare case of PACG in a 32 years old woman with pathologic myopia of -23.0 RLE. The chief complaint was blurring of the right eye three months before the visit. Right eye Intraocular Pressure (IOP) was $30 \mathrm{mmHg}-38 \mathrm{mmHg}$ despite treatment with three intra-ocular pressure-lowering agents. Axial length was $32.36 \mathrm{~mm}$ and $31.19 \mathrm{~mm}$ RLE. Anterior chamber depth was $2.36 \mathrm{~mm}$ and 2.60 $\mathrm{mm}$ RLE. Lens thickness was $5.07 \mathrm{~mm}$ and $5.40 \mathrm{~mm}$ RLE. Signs of GON and pathologic myopia were found in both eyes. GON was present asymmetrically (0.9 and 0.6 RLE), with the myopic crescent as well as baring and peripapillary atrophy. The optic disc was slightly tilted with the myopic crescent. There was also a marked sign of retinal pigment epithelium thinning and attenuation along with myopic chorioretinal atrophy. Conclusions: PACG in a young myopic individual is challenging to diagnose because myopia and glaucoma share similar optic nerve head pathology. Comprehensive examinations including gonioscopy, biometry, and OCT may confirm the diagnosis. In the presented case, angle-closure was caused by thick lenses and a shallow anterior chamber, along with excessively long axial length. Primary angle-closure at a young individual with myopic eyes is highly uncommon. Nonetheless, clinicians should always consider glaucoma even in the presence of high axial length and myopic fundus. Keywords: primary angle-closure glaucoma; pathologic myopia
\end{abstract}

\section{Introduction}

Glaucoma is a degenerative eye condition that can lead to blindness, affects $3.5 \%$ of people aged forty and over. Adults over the age of forty are more likely to have Primary Angle-Closure Glaucoma (PACG). Females are more likely than males to develop PACG, and it is also more common in certain ethnic groups, such as Asian populations. PACG is found in around 17.14 million persons over the age of 40 worldwide, with a worldwide incidence of $0.6 \%$ ( $95 \% \mathrm{CI}=0.5-0.8 \%$ ). The global population ${ }^{[1]}$ with PACG is expected to be 23.36 million in 2020 and 32.04 million in 2040, with Asia accounting for more than three-quarters of the PACG population. There are two types of PACG: acute and chronic. Primary Chronic Angle-Closure Glaucoma (PCACG) is more common in older people and is uncommon in young individuals. ${ }^{[2],[3]}$

Diagnosing glaucoma in a highly myopic eye is a challenge as highly myopic fundus shares similar pathologic findings of glaucomatous optic neuropathy. Myopic eyes are linked with a range of visual field abnormalities, and evaluating the cup-to-disc ratio in pathologic myopia with tilted optics will be exceedingly challenging. ${ }^{[4]}$ Careful examination of anterior chamber structure and anterior chamber imaging modalities such as Anterior Segment-OCT is mandatory to define the diagnosis. 


\section{Case presentation}

A32-year-old woman who came to the ophthalmology clinic at Dr. Soetomo General Hospital Surabaya presented with chief complaints of blurring right eye vision for about three months before the visit. The blurring of vision was worsening gradually over time. She was also experience pain and redness in the right eye. The pain was said as worsen with eye movement. The patient was already visit the ophthalmologist clinic with a medication history of twice a day brinzolamide and brimonidine tartrate eye drop, twice a day timolol maleate, and oral acetazolamide. With three intraocularpressure- lowering agents, right eye Intraocular Pressure (IOP) was never below $30 \mathrm{mmHg}$. Visual acuity of the left eye was progressively worsening despite treatment.

At first presentation, the patient's visual acuity was light perception in the right eye with poor light projection and 1/60 in the left eye. The best-corrected visual acuity for the left eye was $4 / 60$ with $S-23,0 \mathrm{D}$ not further improved by pinhole. The patient's IOP was measured by an applanation tonometer mounted on a slit-lamp biomicroscope; it was $31 \mathrm{mmHg}$ in the right eye and $10 \mathrm{mmHg}$ in the left eye. She has worn thick minus lenses since elementary grade. The patient's myopia is still progressing; she worn minus spectacles with $-22 \mathrm{D}$ in both eyes, prescribed four years ago. She was not using any topical or systemic medication for any other ailment.
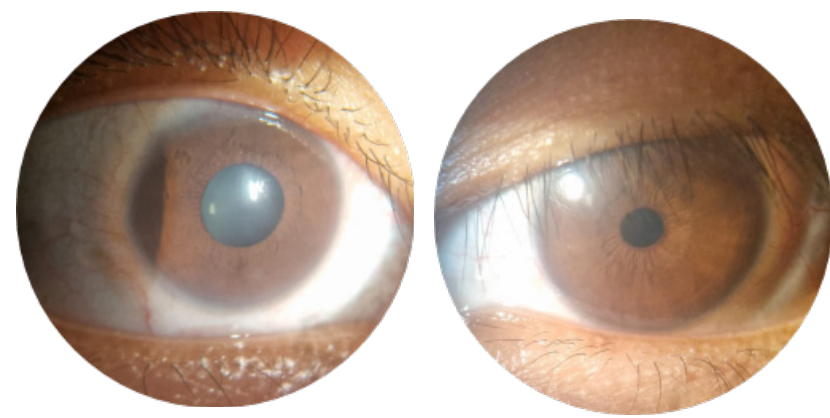

Figure 1. Slit-lamp examination: Diffuse light.

The eyelid, conjunctiva, and cornea did not reveal any noteworthy abnormalities during the anterior segment examination. The anterior chamber showed no sign of inflammation, but it was relatively shallow. The pupil in the right eye was $4 \mathrm{~mm}$ in diameter with minimal light reflex, whereas the pupil in the left eye was $3 \mathrm{~mm}$ and respond immediately to light. On both eyes, a slit-lamp examination revealed a convex iris-lens diaphragm. The Van Herrick technique of anterior chamber evaluation revealed grade 0 , which indicates a closed anterior chamber angle in the right eye, and grade 2, which indicates a narrow anterior chamber angle in the left eye (Figure 1, Figure 2).

A +90D lens was used to examine the posterior region's central retina and other components (Volks, Germany).
In the right and left eyes, the optic disc had a cup-disc ratio of 0.9 and 0.5 , respectively. Other Glaucomatous Optic Neuropathy (GON) signs, such as baring and peripapillary atrophy, were also present in both eyes. In the right eye, the optic disc was slightly tilted with the myopic crescent. There was also a marked sign of retinal pigment epithelium thinning and attenuation, giving the appearance of the tessellated fundus, also known as tigroid fundus. Myopic chorioretinal atrophy was seen in both eyes. Fundus photography was taken for documentation; unfortunately, the fundus photograph of the right eye was blurry due to a small pupil at examination (Figure 9).
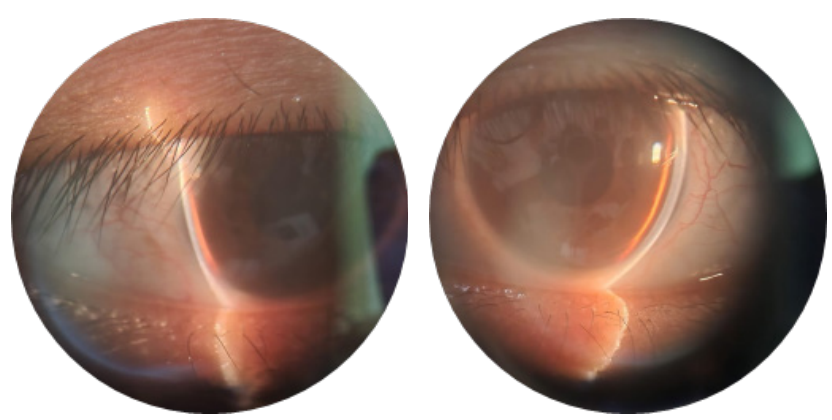

Figure 2. Slit-lamp examination: Van Herrick.

Gonioscopy was performed on both eyes to confirm the diagnosis of angle closure. Only the Schwalbe line was visible at all quadrants of the anterior chamber angle of the right eye. Indentation gonioscopy of the right eye failed to visualize the angle structure, indicating the presence of Peripheral Anterior Synechiae (PAS). Gonioscopy of the left eye shows trabecular meshwork still visible at all quadrants (Figure 3). Anterior-OCT was performed to objectively analyze anterior chamber angle and anterior chamber depth, confirming narrow-angle and shallow anterior chamber, with wide-angle to angle analysis showing right eye scleral spur angle closed and left eye scleral spur angle 9-10 (extreme narrow-angle, Shaffer-system) (Figure 4). Anterior Chamber Depth (ACD) was $1.58 \mathrm{~mm}$ and $1.86 \mathrm{~mm}$, respectively, for the right and left eye. Ancillary testing with HFA and OCT was consistent with comprehensive eye examination confirming GON with a cup-to-disc ratio of $0.98 \mathrm{OD}$ and $0.52 \mathrm{OS}$, correlating Retinal Nerve Fiber Layer (RNFL) and visual field defect.

Axial length, anterior chamber depth, and lens thickness were measured using immersion ultrasound biometry, and the right eye's axial length was $32.36 \mathrm{~mm}$, whereas the left eye was $31.19 \mathrm{~mm}$. The right eye's lens thickness was $5.07 \mathrm{~mm}$, whereas the left eye was 5.40 $\mathrm{mm}$. Compared to AS-OCT, the anterior chamber depth measurement findings utilizing ultrasound biometry were $2.36 \mathrm{~mm}$ and $2.60 \mathrm{~mm}$ for the right and left eyes, respectively. 
The patient was diagnosed with primary angle closure glaucoma and pathologic myopia in both eyes. The intraocular-pressure lowering agent was continued with brinzolamide, brimonidine tartrate eye drop twice a day, timolol maleate twice a day, and oral acetazolamide twice a day. Goniosinechiolysis and peripheral iridectomy were planned for the right eye, and laser peripheral iridectomy subsequently for the left eye.

Table 1. Ultrasound Biometri

\begin{tabular}{|c|c|c|c|c|c|}
\hline & \multicolumn{2}{|c|}{ OD } & \multicolumn{3}{|c|}{ os } \\
\hline & \multicolumn{2}{|c|}{$\mathrm{K} 1: 7.59$} & \multicolumn{3}{|c|}{$\mathrm{K} 1: 7.52$} \\
\hline & \multicolumn{2}{|c|}{ K2 : 7.30} & \multicolumn{3}{|c|}{ K2 : 7.33} \\
\hline & \multicolumn{2}{|c|}{$\mathrm{AXL}: 32.36 \mathrm{~mm}$} & \multicolumn{3}{|c|}{$\mathrm{AXL}: 31.19 \mathrm{~mm}$} \\
\hline & \multicolumn{2}{|c|}{ ACD : $2.36 \mathrm{~mm}$} & \multicolumn{3}{|c|}{ ACD : $2.60 \mathrm{~mm}$} \\
\hline & \multicolumn{2}{|c|}{$\mathrm{LT}: 5.07 \mathrm{~mm}$} & \multicolumn{3}{|c|}{$\mathrm{LT}: 5.40 \mathrm{~mm}$} \\
\hline \multirow[t]{3}{*}{118.0} & -7.0 & 0.06 & \multirow[t]{3}{*}{118.0} & -4.5 & 0.25 \\
\hline & -6.5 & -0.18 & & -4.0 & 0.00 \\
\hline & -6.0 & -0.43 & & -3.5 & -0.25 \\
\hline \multirow[t]{3}{*}{118.1} & -7.0 & 0.04 & \multirow[t]{3}{*}{118.1} & -4.5 & 0.24 \\
\hline & -6.5 & -0.20 & & -4.0 & -0.01 \\
\hline & -6.0 & -0.44 & & -3.5 & -0.26 \\
\hline \multirow[t]{3}{*}{118.4} & -7.0 & -0.01 & \multirow[t]{3}{*}{118.4} & -4.5 & 0.20 \\
\hline & -6.5 & -0.25 & & -4.0 & -0.04 \\
\hline & -6.0 & -0.49 & & -3.5 & -0.29 \\
\hline
\end{tabular}

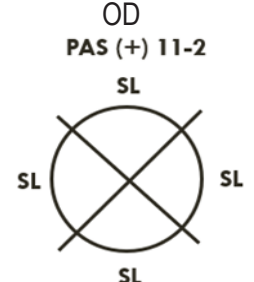

SL

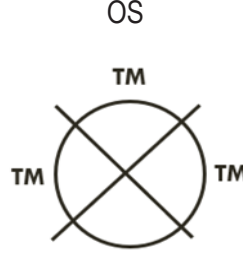

TM
Figure 3. Gonioscopy examination

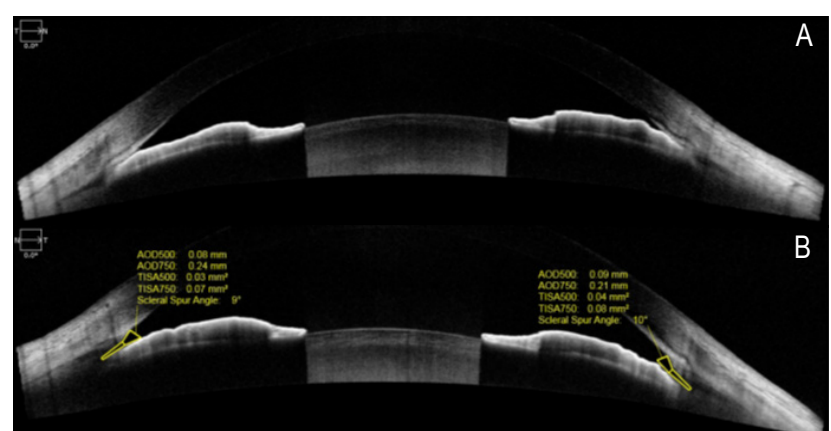

Figure 4. Anterior Segment OCT; (A) Wide-angle to angle right eye shows the closed-angle, and (B) Wide-angle to angle left eye.

\section{Discussion and conclusions}

The case presented in this report is a chronic PACG in a young woman who also has pathologic myopia with extremely long axial length. Chronic PACG at a young age is a challenging case to diagnose and manage, as it needs lifelong follow-up and treatment. Angle-closure is rare in young adults; only isolated cases and small series have been reported. Several studies use the cut-off age of 40 years old to define young age angle-closure. It is used in the cut-off for presbyopia.. ${ }^{[2],[5]}$ Angle-closure in young patients is usually associated with structural or developmental ocular anomalies rather than relative pupillary block. ${ }^{[2]}$

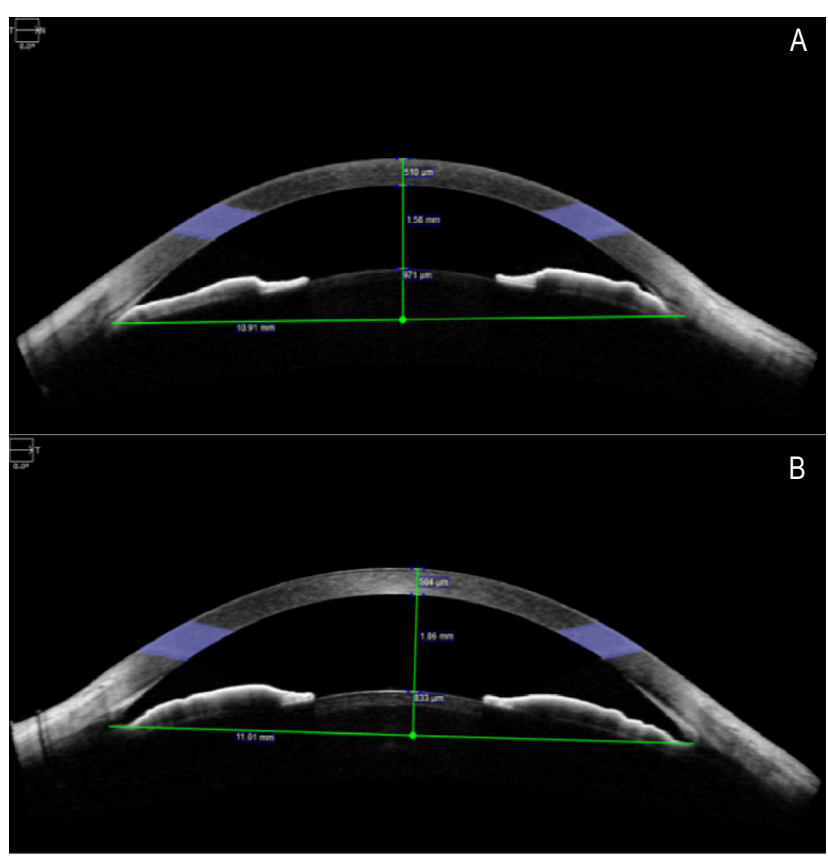

Figure 5. Anterior Segment OCT; (A) Anterior chamber analysis right eye, and (B) Anterior chamber analysis left eye

Myopia has long been thought to carry a risk factor for POAG, whereas hyperopia has a higher prevalence of PACG. Angle-closure can still occur in eyes with severe myopia, according to particular research. Cross-sectional research in Singapore enrolled 427 angle-closure patients, including 143 primary angle-closure suspects, 75 primary angle-closure, 165 with PACG, and 44 acute primary angle-closure patients. Myopia of greater than $0.50 \mathrm{D}$ was found in 94 of the patients who were recruited (22\%). ${ }^{[5]}$ Angle-closure, in this case, was confirmed by gonioscopy examination and Anterior Segment-OCT that shows a closed angle of the right eye with peripheral anterior synechia at 11-2 o'clock and highly narrow (possibly closed-angle) of the left eye.

Eyes that develop Angle-Closure Glaucoma tend to have crowded anterior segments and short axial length. ${ }^{[3],[6]}$ The most important factors leading to the closure of the eye are shallow anterior chamber, thick lens, increased anterior lens curvature, a short axial length, small corneal diameter, and radius curvature. An anterior chamber depth of less than $2.5 \mathrm{~mm}$ is considered shallow enough to predisposes patients with primary angle closure.

In addition, Peripheral Anterior Synechia (PAS) formation increases in an anterior chamber depth of 


\section{\begin{tabular}{ll|l}
\hline Macula Thickness OU: Macular Cube 512x128 & OD $\bigcirc$ & $\bigcirc$ \\
\hline
\end{tabular}}

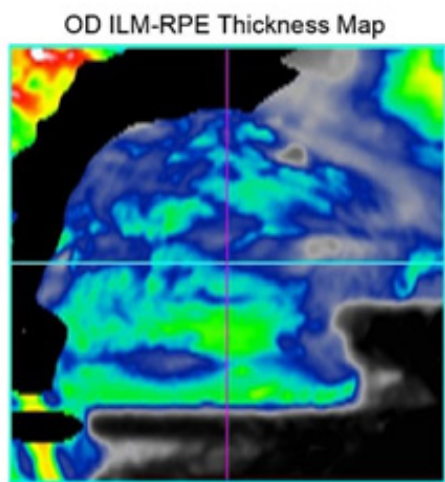

Fovea: Not found

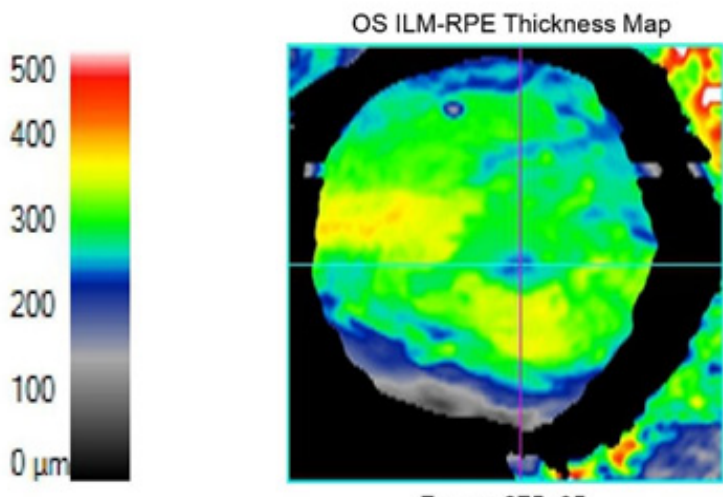

Fovea: 275,65
OD OCT Fundus

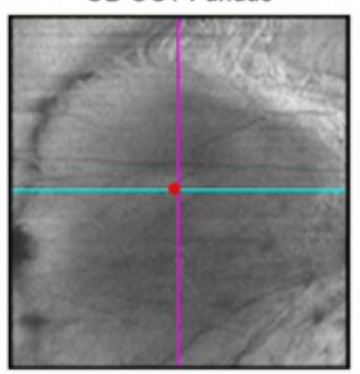

OD ILM-RPE Thickness

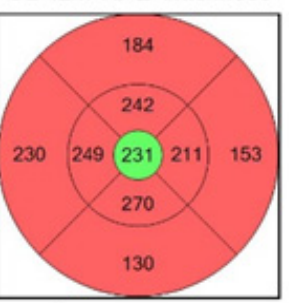

OS ILM-RPE Thickness

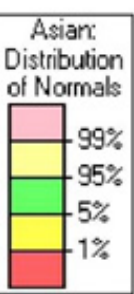

\begin{tabular}{|c|c|c|}
\hline ILM - RPE & OD & OS \\
\hline Thickness Central Subfield $(\mu \mathrm{m})$ & 231 & 280 \\
\hline Volume Cube $\left(\mathrm{mm}^{2}\right)$ & 5.6 & 6.5 \\
\hline Thickness Avg Cube $(\mu \mathrm{m})$ & 156 & 180 \\
\hline
\end{tabular}

OD Horizontal B-Scan BScan: 64
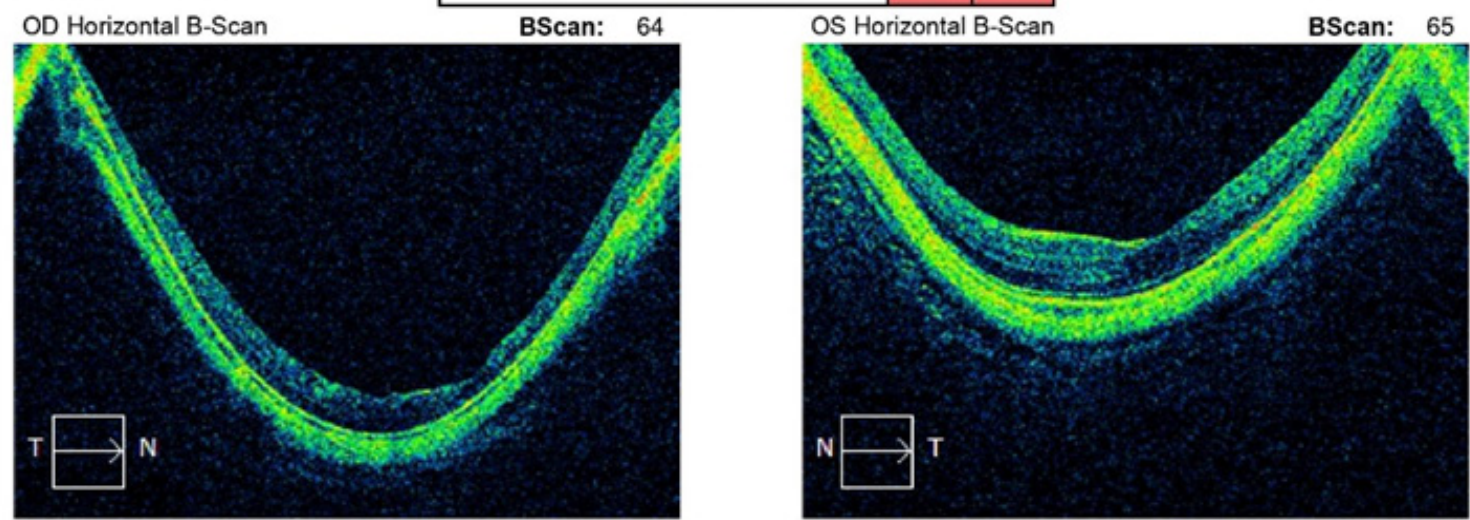

Figure 6. Optical Coherence Tomography (OCT): Macular Thickness analysis showed extremely low average macular cube thickness for the right and the left eye. Horizontal B-scan of the right and the left eye showed posterior staphyloma

less than $2.4 \mathrm{~mm} \cdot{ }^{[3]}$ In this case, the patient was 32 years old with angle-closure was caused by crowded anterior angle, shallow anterior chamber depth, and thick lens. Anterior chamber depth was $1.58 \mathrm{~mm}$ for the right eye and $1.86 \mathrm{~mm}$ for the left eye (obtained using AS-OCT), and lens thickness was $5.07 \mathrm{~mm}$ for the right eye and $5.40 \mathrm{~mm}$ for the left eye (obtained using USG biometry). There were discrepancies in the measurement of anterior chamber depth recorded by Ultrasound Biometry versus Anterior Segment-OCT. ACD obtained by anterior segment OCT measurement is considered more accurate because ultrasound devices can be distorted by operator experiences, US velocity setting, and lower resolution than optical devices. ${ }^{[7],[8]}$
The patient, in this case, has a coexisting pathologic myopia at presentation, which makes fundus examination of glaucomatous optic neuropathy a bit challenging. According to the International Myopia Institute (IMI), pathologic myopia is defined as an excessive axial elongation associated with myopia, which results in structural changes in the posterior segment of the eye, leading to loss of best-corrected visual acuity. Structural changes associated with pathologic myopia including posterior staphyloma, myopic maculopathy, and high myopia-associated optic neuropathy. ${ }^{[9]}$ Glaucomatous optic neuropathy was confirmed by OCT, where the cupdisc ratio was 0.98 OD and 0.52 OS. Macular Ganglion Cell Complex (GCC) parameters of spectral-domain OCT 
serve as a valuable technique for clinically evaluating patients with concomitant glaucoma and high myopia because it was not significantly affected by refractive errors. ${ }^{[10]} \mathrm{A}$ further study conducted by Hsu, Chen, and $\operatorname{Lin}^{[4]}$ in 2015 has shown that the ratio of GCC thickness to macular outer retinal thickness is not influenced by refractive error. They conclude that the ratio of GCC thickness to macular outer retinal thickness provides an accurate diagnosis of glaucoma in highly myopic eyes. The most important point to define glaucoma in myopic eyes is the progression of optic nerve damage. Even in patients with disc and visual field findings that strongly suggest glaucomatous optic neuropathy, confirmation of progression is ultimately necessary to confirm the diagnosis of glaucoma. ${ }^{[11]}$
Furthermore, myopia has been considered a risk factor for the progression of glaucoma. The mechanism of the increased susceptibility of myopia to glaucoma has not yet been fully elucidated. Several theories have been discussed. The theories explaining the association between myopia and glaucoma are the increased stress on the lamina due to the lengthening of the axial length. Cahane and Bartov speculate that even with the same intraocular pressure, the scleral tension in the lamina of myopic eyes is greater than that of eyes with shorter axial lengths. Eye pressure may be higher with thinning of the sclera, which is a common morphological feature of myopia. The increased pressure of the myopia lamina can, in turn, lead to mechanical failure and deformation of the lamina, such as the optic nerve head and fossa

\section{ONH and RNFL OU Analysis:Optic Disc Cube 200x200 OD $\bigcirc$ OS}

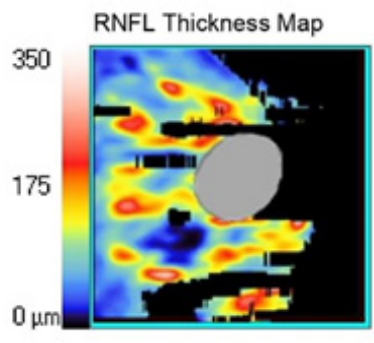

RNFL Deviation Map

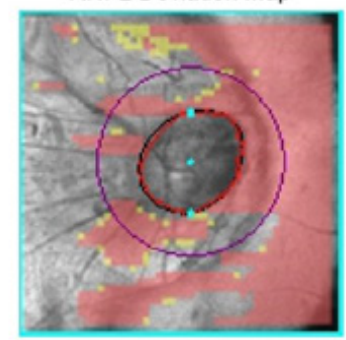

Disc Center(0.15,0.24)mm

Extracted Horizontal Tomogram

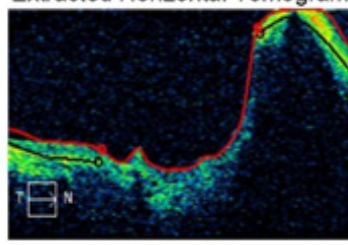

Extracted Vertical Tomogram

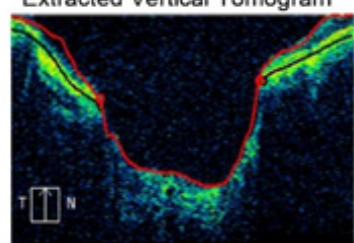

RNFL Circular Tomogram

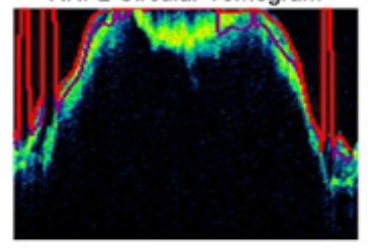

\begin{tabular}{|r|c|c|}
\hline & OD & OS \\
\hline Average RNFL Thickness & $72 \mu \mathrm{m}$ & $101 \mu \mathrm{m}$ \\
\hline RNFL Symmetry & \multicolumn{2}{|c|}{$-28 \%$} \\
\hline Rim Area & $0.30 \mathrm{~mm}^{2}$ & $1.88 \mathrm{~mm}^{2}$ \\
\hline Disc Area & $3.29 \mathrm{~mm}^{2}$ & $2.80 \mathrm{~mm}^{2}$ \\
\hline Average C/D Ratio & 0.96 & 0.53 \\
\hline Vertical C/D Ratio & 0.98 & 0.52 \\
\hline Cup Volume & $1.783 \mathrm{~mm}^{3}$ & $0.043 \mathrm{~mm}^{3}$ \\
\hline
\end{tabular}

Neuro-retinal Rim Thickness

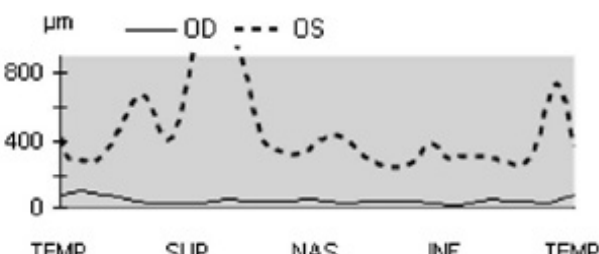

RNFL Thickness
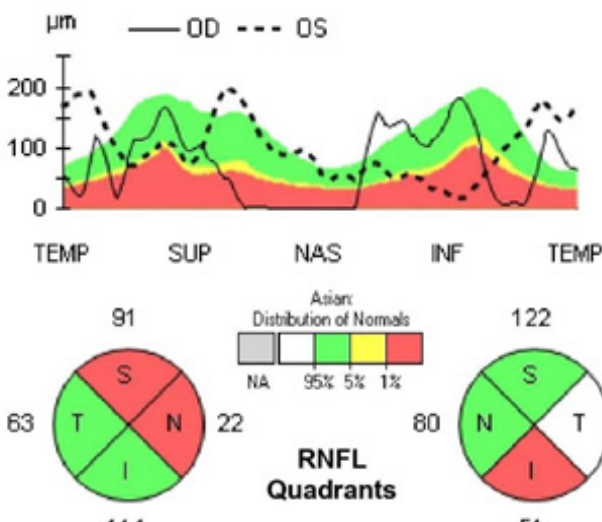

114

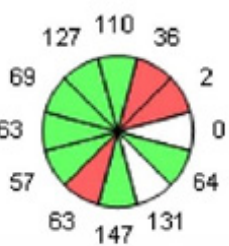

51

$178^{97} 90$

RNFL

Clock

Hours

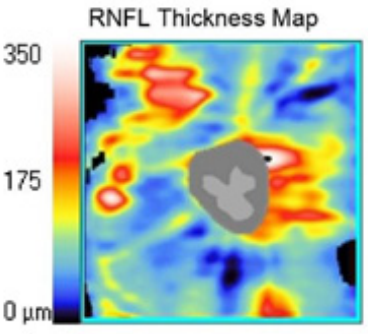

RNFL Deviation Map

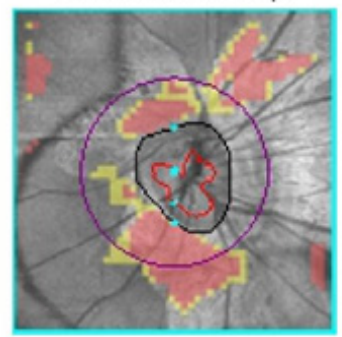

Disc Center $(-0.02,0.01) \mathrm{mm}$ Extracted Horizontal Tomogram

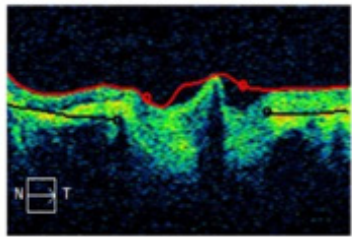

Extracted Vertical Tomogram

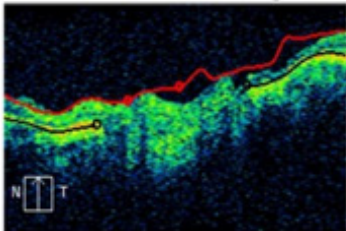

RNFL Circular Tomogram
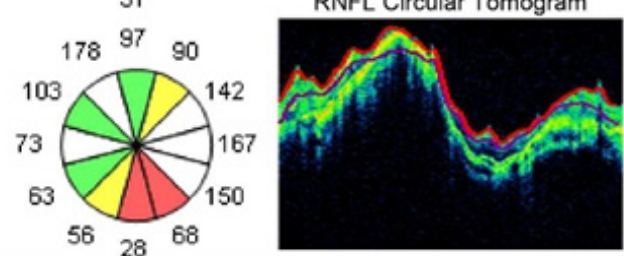

Figure 7. Optical Coherence Tomography (OCT): Optic Nerve Head (ONH) and Retinal Nerve Fiber Layer (RNFL) analysis showed the cup-to-disc ratio of $0.98 \mathrm{OD}$ and $0.52 \mathrm{OS}$ and extremely thin RNFL of the right eye. 
OS Single Field Analysis Central 30-2 Threshold Test

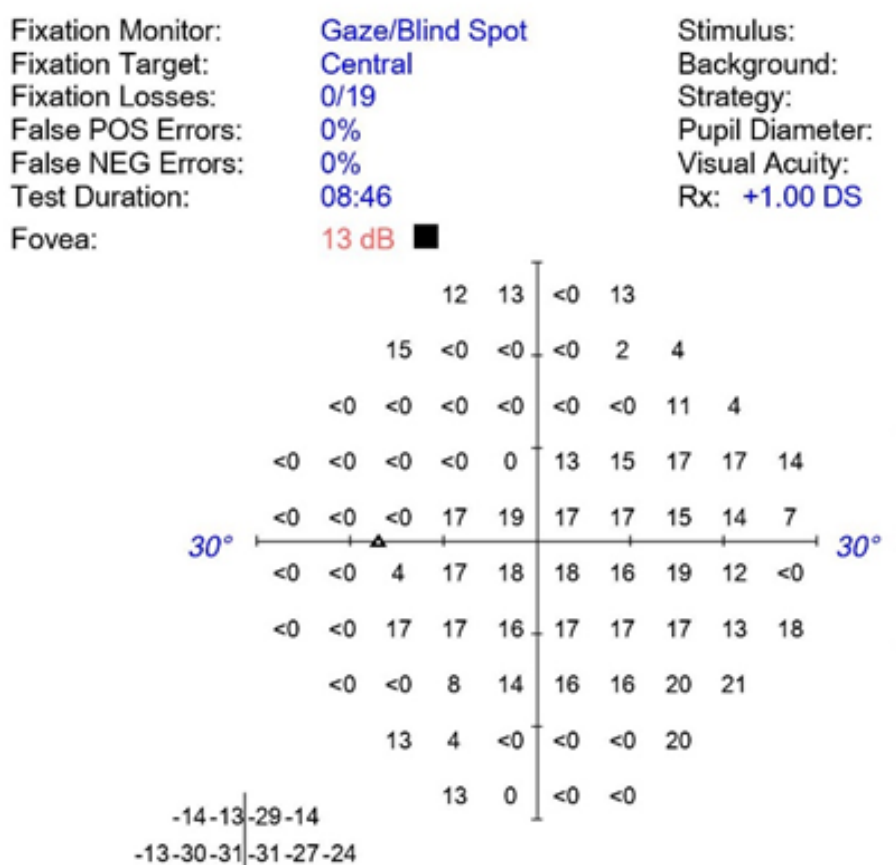

$\begin{array}{lll}\text { III, White } & \text { Date: } & \text { Apr 16, 2021 } \\ \text { 31.5 asb } & \text { Time: } & 11: 28 \text { AM } \\ \text { SITA Standard } & \text { Age: } & 32\end{array}$

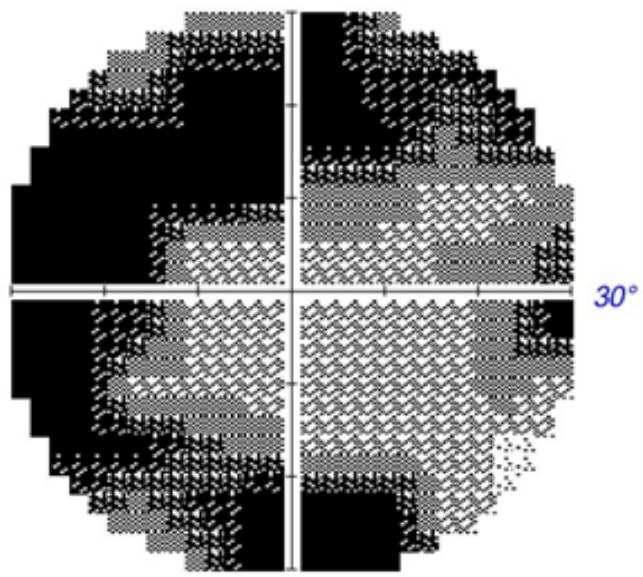

$-31-32-32-33-33-33-19-25$

$-32-32-33-34-33-19-17-15-13-14$ $-32-33 \quad-16-15-17-17-17-17-22$

$-33-33 \quad-16-16-16-17-14-19-31$

$-33-33-15-16-17-16-16-15-18-10$

$-33-33-24-18-16-16-11-9$

$-18-27-33-33-32-9$

$-17-29-31-30$

Total Deviation

$:: \% \mathbf{\square} \%$

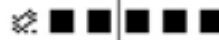

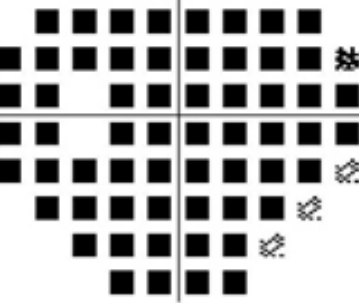

MD Threshold exceeded.

See Total Deviation plot.

$\begin{array}{lll} & \text { GHT: } & \text { Outside Normal Limits } \\ & \text { VFI: } & 36 \% \\ \text { MD30-2: } & -22.05 \mathrm{~dB} \mathrm{P}<0.5 \% \\ \text { Pattern Deviation } & \text { PSD30-2: } & 9.30 \mathrm{~dB} P<0.5 \%\end{array}$

MD Threshold exceeded.

See Total Deviation plot.

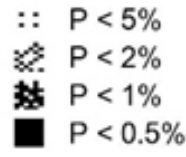

$:: P<5 \%$

音 $\mathrm{P}<1 \%$

$P<0.5 \%$

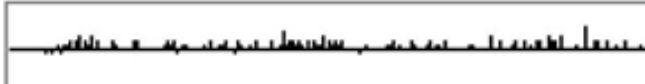

Figure 8. Tunnel-shaped visual field defect obtained from HFA of the left eye.

acquired in the area around the optic nerve head in very myopic eyes. ${ }^{[12]}$

The initial treatments for PACG are to eliminate pupil block and re-open the appositional closure of the anterior chamber angle. Initial treatment options include Laser Peripheral Iridotomy (LPI), surgical peripheral iridectomy, and Argon Laser Peripheral Iridoplasty (ALPI). ${ }^{[13]}$ Iridotomy is necessary to relieve the pupillary block and reduce further synechial angle closure. Without iridotomy, closure of the angle usually progresses and makes subsequent glaucoma more challenging to control. Even in the case of peripheral iridotomy, progressive angle closure can occur, and repeated periodic gonioscopy is very important. Iridotomy with or without longterm intraocular pressure-lowering drugs can control the condition in most patients with chronic PAC. Other commonly used techniques are iridoplasty or lensectomy with or without goniosynechialysis to. Subsequent filtering surgery may be necessary if these measures fail to lower the IOP. ${ }^{[14]}$ Refractive surgery such as precise lens extraction may benefit high myopic eyes because the thick lens predisposes patients to angle-closure glaucoma. Lens extraction may have an IOP-lowering effect that ranges between 2 and $6 \mathrm{mmHg}$ (up to $12 \mathrm{mmHg}$ in one study) in eyes with PACG. ${ }^{[15]}$ Removing a thickened and anteriorly positioned lens leads to restoring deeper 
angle configuration, which may be advantageous in eyes with PACG and leads to a significant IOP reduction. ${ }^{[15],[16]}$

Weplanned surgicaliridotomy with goniosynechialysis of the left eye for the initial treatment and prophylactic laser peripheral iridotomy of the left eye, along with careful observation of disease progression. Refractive surgery with precise lens extraction has been considered but is too risky to be immediately done because of the extremely shallow anterior chamber.
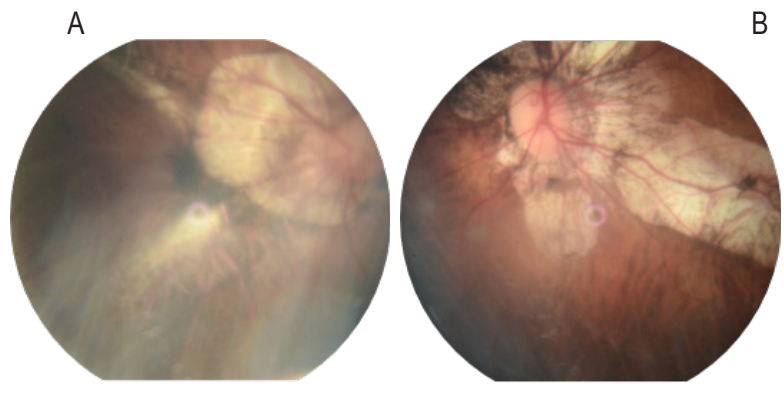

Figure 9. Fundus Photography of (A) Right Eye, fundus photograph was blurry due to small pupil. IOP of the right eye was never below $30 \mathrm{mmHg}$ that we were unable to dilate the pupil for better documentation; (B) Left eye fundus photograph shows marked retinal pigment epithelium thinning and attenuation, giving the appearance of the tessellated fundus, also known as the tigroid fundus, as well as myopic chorioretinal atrophy.

Primary angle-closure at a young age with myopic eyes is hugely uncommon. This case represents a rare finding of shallow anterior chambers with thick lenses predisposing high axial myopic eyes to angle closure. Clinicians should always consider the possibility of angleclosure glaucoma even in the presence of high axial length and myopic fundus. History taking and examination may guide to the definitive diagnosis and treatment.

\section{References}

[1] Zhang N, Wang J, Chen B, Li Y, Jiang B. Prevalence of primary angle closure glaucoma in the last 20 Years: A meta-analysis and systematic review. Front Med 2021;7:1-10. https://doi. org/10.3389/fmed.2020.624179.

[2] Ritch R, Chang BM, Liebmann JM. Angle closure in younger patients. Ophthalmology 2003;110:1880-1889. https://doi. org/10.1016/S0161-6420(03)00563-3.

[3] Girkin CA, Bhorade AM, Crowston JG, Giaconi JA, Medeiros FA, Sit AJ, et al., editors. Angle-Closure Glaucoma. Glaucoma 2019-2018 Basic Clin. Sci. Course, San Fransisco: American Academy of Ophthalmology; 2019, p. 143-178.

[4] Hsu CH, Chen RI, Lin SC. Myopia and glaucoma: Sorting out the difference. Curr Opin Ophthalmol 2015;26:90-95. https://doi.org/10.1097/ICU.0000000000000124.

[5] Yong KL, Gong T, Nongpiur ME, How AC, Lee HK, Cheng L, et al. Myopia in Asian subjects with primary angle closure: Implications for glaucoma trends in East Asia. Ophthalmology 2014;121:1566-1571. https:/doi. org/10.1016/j.ophtha.2014.02.006.
[6] Wang T-H, Hsieh J-W, Hung P-T. Anatomy and Biometry. In: Hong C, Yamamoto T, editors. Angle Clos. Glaucoma, Amsterdam: Kugler Publications; 2007, p. 19-28.

[7] Nemeth G, Vajas A, Tsorbatzoglou A, Kolozsvari B, Modis L, Berta A. Assessment and reproducibility of anterior chamber depth measurement with anterior segment optical coherence tomography compared with immersion ultrasonography. J Cataract Refract Surg 2007;33:443-447. https://doi.org/10.1016/j.jcrs.2006.11.013.

[8] Rashid H, Sharif AO, Shipu ZR, Ghosh D, Akhter S, Priyadarshini F, et al. Comparative study between optical and ultrasonic measurement of axial length and anterior chamber depth in normal, long and short eyes. J Shaheed Suhrawardy Med Coll 2020;11:147-154. https://doi. org/10.3329/jssmc.v11i2.48968.

[9] Ohno-matsui K, Wu P, Yamashiro K, Vutipongsatorn K, Fang Y, Ming C, et al. IMI Pathologic Myopia 2021:8-10.

[10] Shoji T, Nagaoka Y, Sato H, Chihara E. Impact of high myopia on the performance of SD-OCT parameters to detect glaucoma. Graefe's Arch Clin Exp Ophthalmol 2012;250:1843-1849. https://doi.org/10.1007/s00417-012-1994-8.

[11] Kim YY. Patophysiology of Angle-Closure Glaucoma. In: Hong C, Yamamoto T, editors. Angle Clos. Glaucoma, Amsterdam: Kugler; n.d.

[12] Miki A, Ikuno Y, Asai T, Usui S, Nishida K. Defects of the lamina cribrosa in high myopia and glaucoma. PLoS One 2015;10:112. https://doi.org/10.1371/journal.pone.0137909.

[13] Tanner L, Gazzard G, Nolan WP, Foster PJ. Has the EAGLE landed for the use of clear lens extraction in angle-closure glaucoma? And how should primary angle-closure suspects be treated? Eye 2020;34:40-50. https://doi.org/10.1038/ s41433-019-0634-5.

[14] Prum BE, Herndon LW, Moroi SE, Mansberger SL, Stein JD, Lim MC, et al. Primary angle closure. Ophthalmology 2016;123:P1-40. https://doi.org/10.1016/j. ophtha.2015.10.049.

[15] Baig N, Kam K-W, Tham CCY. Managing primary angle closure glaucoma - The role of lens extraction in this Era. Open Ophthalmol J 2016;10:86-93. https://doi.org/10.217 4/1874364101610010086.

[16] Eid TM. Primary lens extraction for glaucoma management: A review article. Saudi J Ophthalmol 2011;25:337-345. https://doi.org/10.1016/j.sjopt.2011.07.004. 